\title{
QUEEN'S
UNIVERSITY
BELFAST
}

\section{Modulation Insensitive PLL for Tracking Antenna Applications}

Buchanan, N. B., \& Fusco, V. F. (2015). Modulation Insensitive PLL for Tracking Antenna Applications. Microwave and Optical Technology Letters, 57(6), 1286-1289. https://doi.org/10.1002/mop.29073

Published in:

Microwave and Optical Technology Letters

Document Version:

Peer reviewed version

Queen's University Belfast - Research Portal:

Link to publication record in Queen's University Belfast Research Portal

Publisher rights

(C) 2015 Wiley Periodicals, Inc.

This is the peer reviewed version of the following article: Buchanan, NB \& Fusco, VF 2015, 'Modulation Insensitive PLL for Tracking Antenna Applications' Microwave and Optical Technology Letters, vol 57, no. 6, pp. 1286-1289., 10.1002/mop.29073, which has been published in final form at http://onlinelibrary.wiley.com/doi/10.1002/mop.29073/abstract. This article may be used for non-commercial purposes in accordance With Wiley Terms and Conditions for self-archiving

\section{General rights}

Copyright for the publications made accessible via the Queen's University Belfast Research Portal is retained by the author(s) and / or other copyright owners and it is a condition of accessing these publications that users recognise and abide by the legal requirements associated with these rights.

Take down policy

The Research Portal is Queen's institutional repository that provides access to Queen's research output. Every effort has been made to ensure that content in the Research Portal does not infringe any person's rights, or applicable UK laws. If you discover content in the Research Portal that you believe breaches copyright or violates any law, please contact openaccess@qub.ac.uk. 


\title{
Modulation Insensitive PLL for Tracking Antenna Applications
}

\author{
N.B. Buchanan, V.F. Fusco
}

The Institute of Electronics, Communications and Information Technology (ECIT), Queen's University Belfast, Northern Ireland Science Park, Queen's Road, Queen's Island, Belfast, BT3 9DT, Tel +44 2890971721, Fax +44 289097 1702, n.buchanan@ecit.qub.ac.uk

\begin{abstract}
This paper shows practical results of a self tracking receiving antenna array employing a new phase locked loop (PLL) tracking configuration. The PLL configuration differs from other architectures, as it has the new feature of being able to directly track phase modulated signals without requiring an additional unmodulated pilot carrier to be present. The PLLs are used within the antenna array to produce a constant phase intermediate frequency (IF) for each antenna element. These IF's can then be combined in phase, regardless of the angle of arrival of the signal, thus utilising the antennas array factor. The papers main focus is on the phase jitter performance of the modulation insensitive PLL carrier recovery when tracking phase modulated signals of low signal to noise ratio. From this analysis, it is concluded that the new architecture, when optimally designed, can produce phase jitter performance close to that of a conventional tracking PLL.
\end{abstract}

\section{Introduction}

Phase locked loops are extensively used in carrier recovery applications [1]. They have the advantage of being able to acquire a weak signal and produce a carrier which tracks the phase of the signal being recovered. Recently it has been shown [2] that tracking PLLs are an invaluable addition to phased array self-tracking antennas for applications such as satellite. The concept of tracking phased array antennas using phased locked loops has been proposed in [3] wherein the received signals can be optimally combined in phase. This architecture was practically shown in [4] with the additional feature of a conjugated phase signal being sent back in the same direction as the received signal.

One of the major difficulties in using a classical tracking PLL architecture to track signals in modern communications scenarios is that the signals to be tracked usually contain some complex modulation scheme involving rapid changes to both the phase and amplitude of the signal, preventing a PLL circuit stably locking to the signal.

\section{Conventional Tracking PLL with QPSK signal}

The architecture of a classical tracking PLL is shown in Fig.1, here the phase detector is a multiplier type, with a reference signal $V_{\text {REF }}(\cos \omega t)$. We assume a QPSK modulated signal is applied to the antenna input. This is down converted via the mixer, filtered, and fed to the phase detector. We make the assumption that the phase variation, due to the bit rate of the phase modulation, is considerably faster than the time constant of the PLL loop filter, this will give a signal at the output of the loop filter of the form 


$$
\frac{V_{R E F} V_{1}}{2}\left[\cos \left(\varnothing-(2 n-1) \frac{\pi}{4}\right)\right]
$$

Where each of the four QPSK phase states is denoted by $n=1,2,3,4$, and $\phi$ is the phase term due to the direction of arrival of the signal to the antenna. For QPSK modulation with probability of $n=1,2,3,4$ of 0.25 , Equation 1 becomes equal to 0 , regardless of the value of $\phi$. Therefore the angle of arrival information cannot be determined and the PLL will be unable to attain a stable locked condition. This renders the conventional PLL unsuitable for tracking phase modulated signals such as QPSK.

Other PLL structures can be used to track phase modulated signals, such as QPSK. The multiply filter divide architecture [5] multiplies the QPSK signal by 4, making the $90^{\circ}$ phase transitions, effectively $0^{\circ}$. This multiplication can be challenging when operating with low $\mathrm{S} / \mathrm{N}$ ratios. Another architecture, the Costas loop [6], uses a more complex phase detector arrangement, which is difficult to realise directly at microwave frequencies. The architectures in $[5,6]$ also are unable to distinguish which instantaneous $90^{\circ}$ transition to lock to. This creates a random phase ambiguity between elements in a phased array, which would require complicated synchronisation methods to overcome.

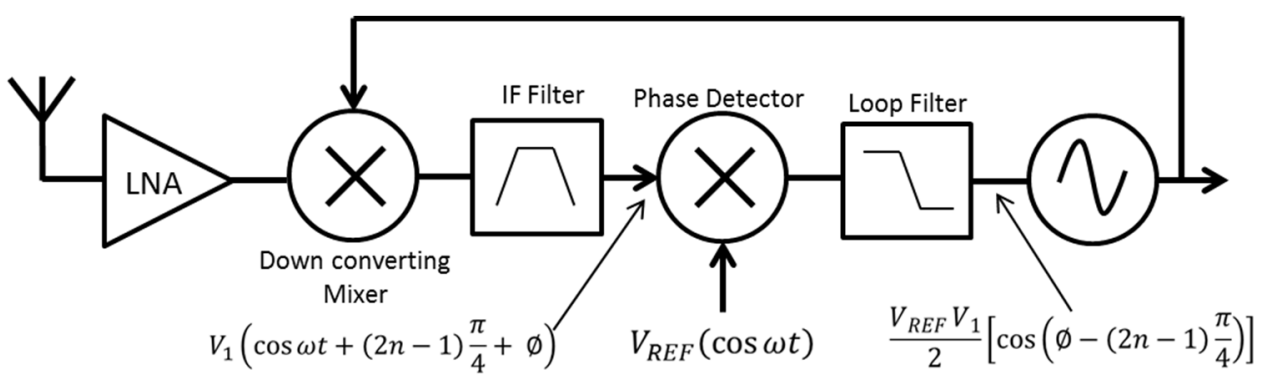

Fig. 1 Conventional tracking PLL for constant phase IF

\section{Modulation Insensitive Tracking PLL Architecture}

The modulation insensitive PLL (MIPLL) now described is intended to offer a simple, yet effective means of tracking the incoming signal phase across an array, such that the received signals can be optimally combined. Fig. 2 shows the operating principle of the modulation insensitive tracking PLL (MIPLL). Assume a modulated signal, (QPSK), is received at the two antenna inputs in Fig. 2, these signals have amplitudes of $V_{1}, V_{2}$ and signal $V_{2}$ arrives with a phase $\phi$, due to the angle of arrival. After down conversion the instantaneous signal produced at the output of the loop filter is

$$
\frac{V_{1} V_{2}}{2}(\cos \varnothing)
$$

which can be used to lock the PLLs in the array. 
Assuming that an exclusive OR type of phase detector is employed, e.g. [7], then at stable lock, with the phase detector output at mid-range, $V_{1}$ and $V_{2}$ at the inputs to the phase detector (Fig. 2) will, be in quadrature. Therefore the output signals for each of the array elements will be optimally summed in-phase when added via a $90^{\circ}$ power combiner (Fig. 2). This allows in phase combination on receive, thus optimally utilising the antenna array factor in self tracking receive mode.

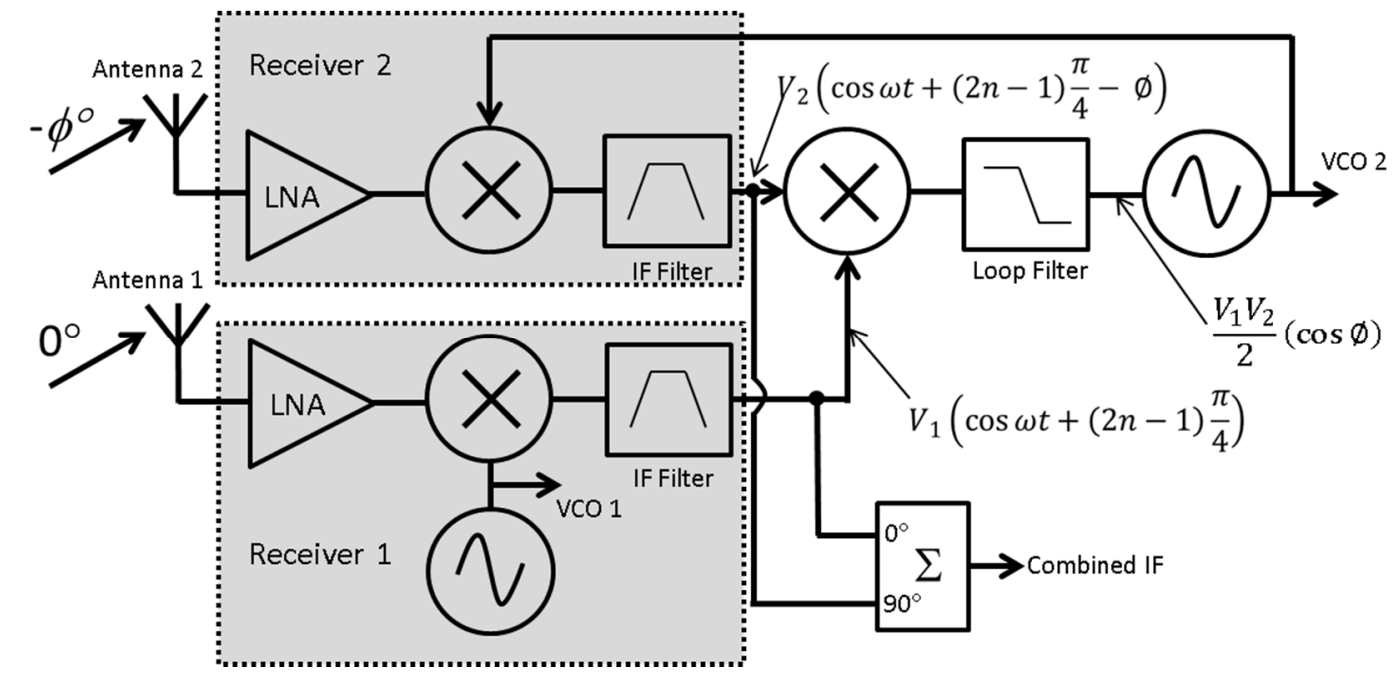

Fig. 2 Modulation Insensitive PLL (MIPLL), showing instantaneous signal expressions

\section{Comparison of MIPLL and conventional PLL when tracking weak signals}

\section{(a) Conventional PLL}

When a PLL is required to track a weak signal in a satellite communications scenario, the noise on the signal will be translated to phase jitter on the output of the PLL. An extensive analysis of phase jitter relating to the conventional PLL was shown in [8] and the phase jitter given by

$$
\text { RMS Phase Jitter } r_{\text {conv }}(d B R a d s)=\frac{1}{2}\left[10 \log \left(B_{L F c o n v}\right)-S(d B)+10 \log (\mathrm{kT})+\mathrm{F}(\mathrm{dB})\right]
$$

Equation 3

Equation 3 shows that RMS phase jitter is not dependant on IF filter bandwidth, only the loop filter bandwidth when $B_{\mathrm{IF}}>2 \mathrm{~B}_{\mathrm{LF}}$ (shown in [8]). From [9] it can be shown that the RMS phase jitter is inversely proportional to the $\mathrm{S} / \mathrm{N}$ ratio at the output of the loop filter.

\section{(b) Modulation Insensitive PLL (MIPLL)}

In the MIPLL the reference signal is fed from the down converted signal from Antenna 1, Fig. 2, which contains both the wanted signal and noise. This reference signal is of the form:

$$
V_{1}(\cos \omega t)+V_{n}(t) \cos \left(\omega t+\emptyset_{n}(t)\right)+V_{n 1}(t) \cos \left(\omega t+\emptyset_{n 1}(t)\right)
$$


and the signal from Antenna 2 (Fig. 2) in the array is fed to the phase detector is of the form:

$$
V_{2}(\cos \omega t-\emptyset)+V_{n}(t) \cos \left(\omega t+\emptyset_{n}(t)-\emptyset\right)+V_{n 2}(t) \cos \left(\omega t+\emptyset_{n 2}(t)\right)
$$

Equation 5

Where $V_{n}(t)$ and $\phi_{n}(t)$ are the amplitude and phase terms of the coherent noise, $N$, added during propagation. The angle of arrival at the antenna yields phase delay, $\phi$, while $V_{n 1}(t), V_{n 2}(t), \phi_{n 1}(t)$ and $\phi_{n 2}(t)$ are the amplitude and phase terms of the noise generated in the individual receivers. We now assume that the signal amplitudes at antennas 1 and 2 are equal and write: $V_{1}=V_{2}=V_{s}$. We also make the assumption that each receiver has an equal level of noise, therefore $V_{n 1}=V_{n 2}=V_{n r}$ where $V_{n r}$ is the noise level at each receiver. The most significant noise level is assumed to be that of the receiver, which means that that $V_{n r} \gg V_{n}$. With these assumptions in mind, it is then possible to approximate the $\mathrm{S} / \mathrm{N}$ at the output of the MIPLL phase detector from the product of Equation 4 and Equation 5, as:

$$
\frac{S}{N}=\frac{V_{s}^{2}}{V_{s} V_{n r}+V_{n r}^{2}}
$$

Equation 6

which upon using Equation 3 suggests that for the MIPLL RMS phase jitter is proportional to the inverse of the $\mathrm{S} / \mathrm{N}$ ratio at the loop filter output:

$$
\text { RMS Phase Jitter }{ }_{\text {mipll }}=-\frac{1}{2}\left[20 \log (S)-10 \log \left(\mathrm{S}+\mathrm{kTF} B_{I F}\right)-10 \log (\mathrm{kT})-\mathrm{F}(\mathrm{dB})-10 \log \left(B_{L F}\right)\right]
$$

Equation 7

Equation 7 indicates that RMS phase jitter is dependent on both the IF filter bandwidth and the loop filter bandwidth. Therefore, reducing the loop filter bandwidth alone, as is possible in the conventional PLL, will not optimally increase the $\mathrm{S} / \mathrm{N}$ ratio at the output of the MIPLL loop filter, since this also requires the IF filter bandwidth to be as narrow as possible. This means that, for the MIPLL, the IF bandwidth should be approximately equal to the bandwidth of the signal being tracked, in order to allow maximum signal power to pass, whilst also filtering as much noise as possible. In the case of Inmarsat BGAN [10] this could be in the region of $10 \mathrm{KHz}$ to more than 200 $\mathrm{KHz}$ depending on the type of signal (e.g. global beam, spot beam) and bit rate.

\section{Experimental results}

\section{(a) Modular PLL arrangement for MIPLL/conventional PLL comparison}

The tracking PLL was first measured in a non-optimised modular configuration, where the component parts could be rearranged into a conventional PLL (Fig. 1) or MIPLL (Fig. 2). This allowed direct measured comparison between the two configurations. The modular PLL had a receiver noise figure of $10 \mathrm{~dB}$, a loop filter bandwidth of $1 \mathrm{KHz}$ and an IF filter bandwidth of $80 \mathrm{KHz}$. The VCO (a $26 \mathrm{MHz}$ VCXO with a PLL multiplier to $156 \mathrm{MHz}$ ), had an estimated residual phase jitter of $10^{\circ} \mathrm{RMS}$.

The input signal level to the each PLL configuration was varied and the RMS phase jitter produced between the two $156 \mathrm{MHz}$ VCO outputs measured. The results are shown in Fig. 3, along with 
calculated results obtained from Equation 3 and Equation 7 for the conventional PLL and MIPLL configurations respectively. The calculations also include the $10^{\circ}$ residual VCO phase jitter from the VCO. The results show that for moderate signal levels $(>-110 \mathrm{dBm})$ the phase jitter performance is very similar for the conventional PLL and the MIPLL. The difference becomes more apparent when the signal reduces $(<-120 \mathrm{dBm})$. At this point it is seen that the phase jitter of the MIPLL increases more rapidly than the conventional $\mathrm{PLL}$, this is due to the relationship for the $\mathrm{S} / \mathrm{N}$ ratio at the phase detector output (Equation 6 ) which degrades the $\mathrm{S} / \mathrm{N}$ at the phase detector more rapidly when input signal $\mathrm{S} / \mathrm{N}$ ratio is low.

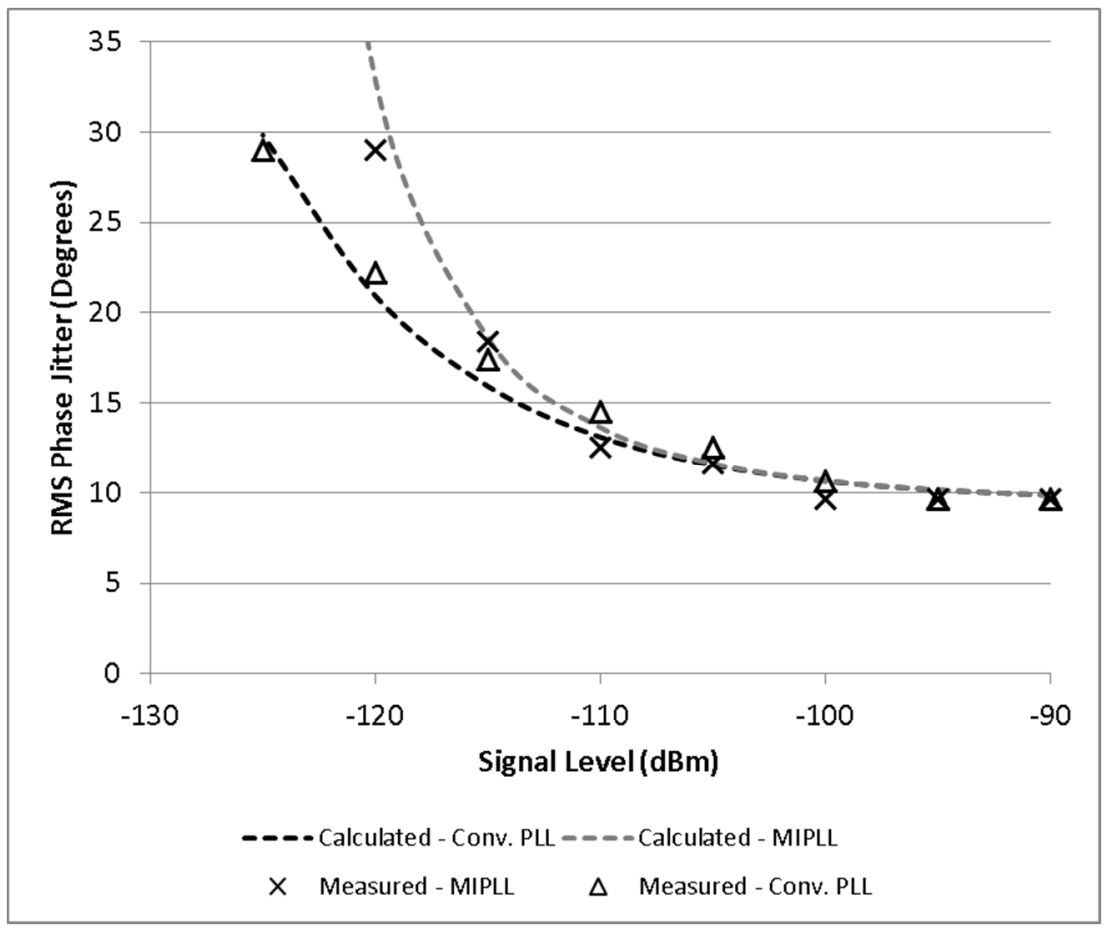

Fig. 3 Phase jitter results for MIPLL compared to conventional PLL

\section{(b) Optimised arrangement for MIPLL communications satellite reception}

Fig. 3 shows that modulated signal levels down to a level of $-120 \mathrm{dBm}$ can be tracked with a nonoptimised configuration. Signal levels from communications satellites (e.g. Inmarsat BGAN [10]) can be as low as $-130 \mathrm{dBm}$. The MIPLL was next designed as an optimised, single PCB arrangement (Inset, Fig. 4) to track a global beam signal from the Inmarsat satellite, frequency $(1.5-1.6 \mathrm{GHz})$, with signal level at the antenna in the region of $-130 \mathrm{dBm}$ for Inmarsat BGAN [10], and continuously modulated with QPSK at a bit rate of $8.4 \mathrm{ksym} / \mathrm{s}$.

A high $\mathrm{Q}$ temperature compensated crystal oscillator (TCVCXO) is used as the VCO, with a residual phase jitter of $5^{\circ} \mathrm{RMS}$. The IF filter in the receiver was set to a $10 \mathrm{KHz}$ bandwidth, to be a close match to the bandwidth of the 8.4ksym/s QPSK signal (Inmarsat BGAN Global beam [10]). The loop filter used a second order active filter, with a cut off frequency of $100 \mathrm{~Hz}$.

The results of Fig. 4 show a low level of phase jitter at signal levels of $-130 \mathrm{dBm}$, producing a phase jitter of $\angle 15^{\circ} \mathrm{RMS}\left(10^{\circ} \mathrm{RMS}\right.$ above the $5^{\circ} \mathrm{RMS}$ residual phase jitter from $\left.\mathrm{VCO}\right)$, c.f. the conventional PLL calculated result of $<12^{\circ} \mathrm{RMS}$, i.e. a difference of only $3^{\circ} \mathrm{RMS}$. Note that only a calculated result 
is shown for the conventional PLL, since the conventional PLL could not track modulated signals and the single $\mathrm{PCB}$ arrangement was not reconfigurable to this arrangement.

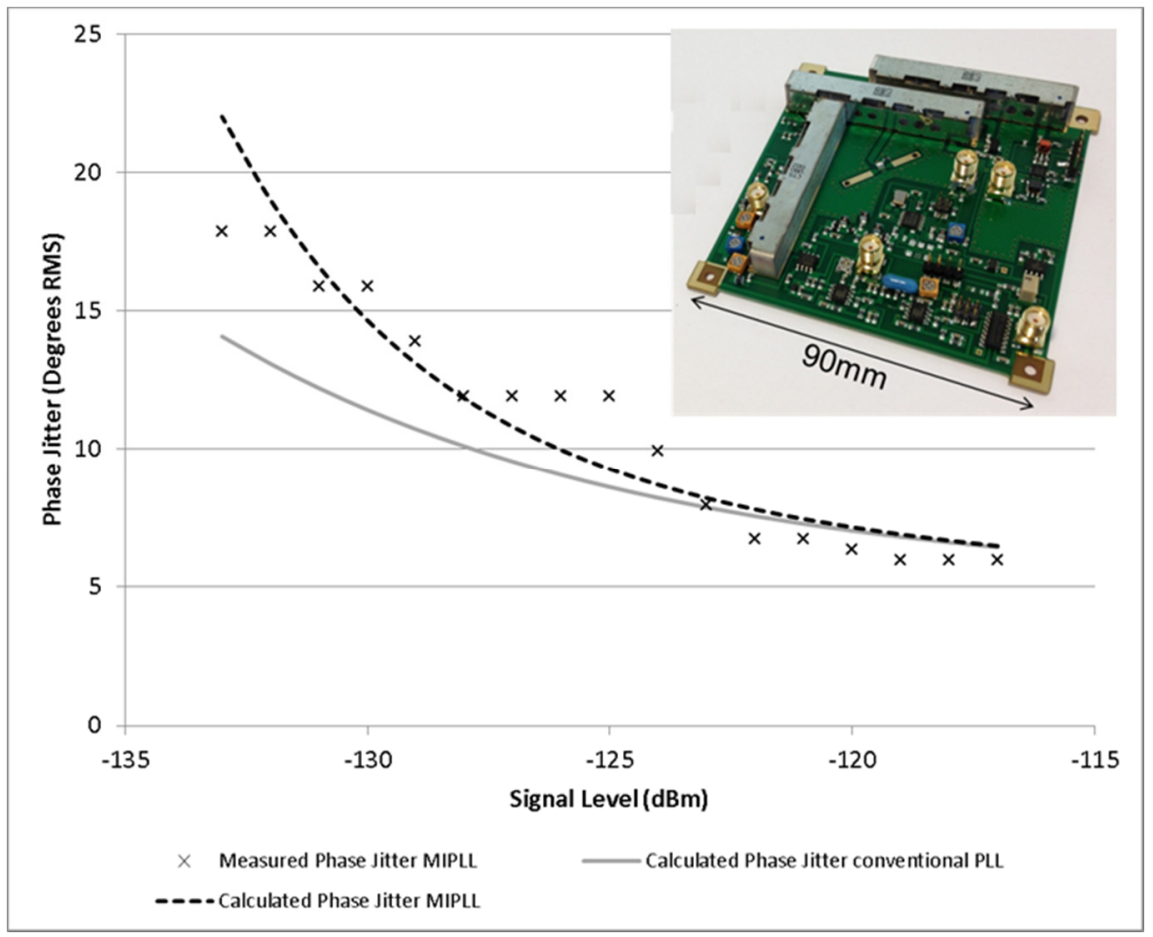

Fig. 4 Phase jitter results for single PCB optimised MIPLL

\section{(c) Phase tracking of Inmarsat global beam with two element array}

With the optimised MIPLL arrangement of Fig. 2, in a two element patch antenna array, the array was rotated in azimuth and the relative phase between the two $156 \mathrm{MHz}$ VCXO signals measured. Fig. 5 shows that phase tracking readily occurs between $+/-40^{\circ}$ azimuth rotation, well within the useable beam width of patch antennas. Also shown in Fig. 5 is a reference calculation of relative signal phase Vs azimuth angle, assuming isotropic antennas. 


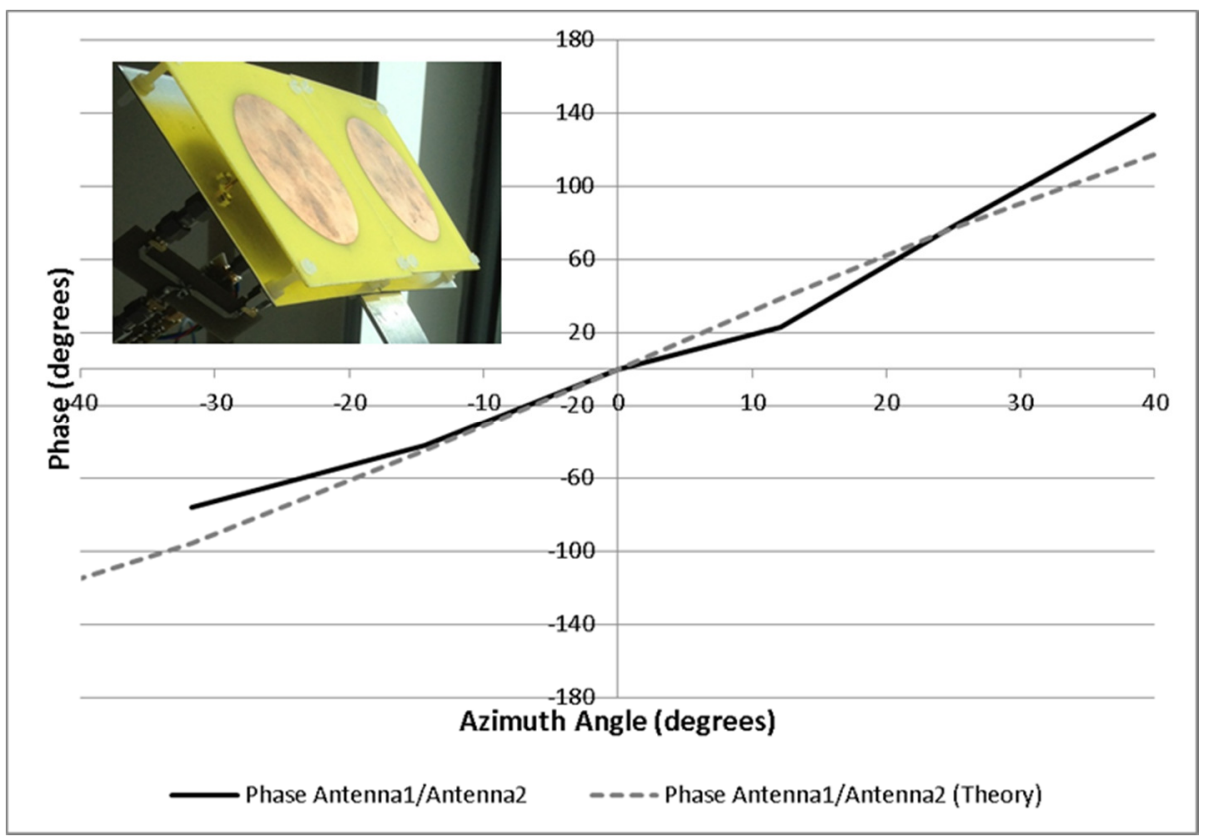

Fig. 5 Phase tracking result for 2x MIPLL/antenna modules tracking the Inmarsat Global beam in Azimuth

\section{Conclusions}

This paper reports the first practical results for a new, modulation insensitive, tracking phase locked loop arrangement. Analysis has shown that performance is only marginally degraded at low $\mathrm{S} / \mathrm{N}$ as compared to a conventional tracking PLL which cannot track modulated signals. The new PLL architecture has been experimentally demonstrated to be able to track very weak signals from a communications satellite in $\mathrm{L}$ band making it suitable for use as a tracking receiver in low cost satellite tracking terminals.

\section{References}

1. F. M. Gardner, Phaselock Techniques, John Wiley \& Sons Inc., New York, NY, 1979

2. Buchanan, N, Fusco, V, Van Der Vorst, M., Williams, N., Winter, C., "New Retrodirective Antenna Techniques for Mobile Terminal Applications" 32nd Antenna Workshop, ESA/ESTEC, Noordwijk, The Netherlands, 5-8 October 2010

3. Bickmore, Robert W., "Adaptive antenna arrays," Spectrum, IEEE , vol.1, no.8, pp.78,88, Aug. 1964

4. N. Buchanan, V. Fusco, M. Van Der Vorst, "A High Performance Analogue Retrodirective Phase Conjugation Circuit with RX Array Factor Combination Ability" IMS 2011, Baltimore

5. J. Mark Steber "PSK Demodulation: Part 1", WJ Tech Notes 1984, Vol. 11 No. 2 March/April 1984

6. John P. Costas "Introduction to Synchronous Communications", Proceedings of the IEEE, December 1956

7. 74HC4046 Phase-locked-loop with VCO, Data Sheet, Philips Semiconductor, Nov 251997

8. P. V. Brennan, Phase-locked loops: principles and practice, London: Macmillan Press Ltd., 1996. 
9. P Lian, "Improving tracking performance of PLL in high dynamic applications" MSc Thesis, University of Calgary, 2004

10. Inmarsat BGAN, http://www.inmarsat.com/service/bgan/ (Accessed on 3-7-2014) 